\title{
Incidência de parto cesárea em uma maternidade no município de Porto Velho - RO em 2017
}

\author{
Incidence of cesarean delivery in a maternity ward in the municipality of Porto Velho - RO \\ in 2017
}

Incidencia de cesárea en una sala de maternidad del municipio de Porto Velho - RO en 2017

Jessika Nauama Silva Pinto ${ }^{*}$, Alessandra da Silva Dantas ${ }^{1}$, Josimeire Souza de Oliveira Andrade $^{1}$, Tathiane Souza de Oliveira ${ }^{1}$, Francisco Mateus Lima da Silva ${ }^{1}$, Jucelino Santos da Silva².

\section{RESUMO}

Objetivo: Descrever a incidência de partos cesárea, comparando aos partos eutócicos realizados em uma Maternidade Municipal de Porto Velho Rondônia no ano de 2017. Métodos: Trata-se de uma pesquisa descritiva e quantitativa de caráter transversal, a partir de registros de mulheres submetidas aos partos cesáreas e eutócicos. Os dados foram tabulados considerando as variáveis: quantitativo de partos eutócicos e cesarianas, faixa etária das gestantes e critérios de indicação médica para o parto cesárea. Os dados obtidos foram analisados mediante utilização do Software Excel 2016, onde os resultados foram expressos por meio de tabela e gráficos. A pesquisa teve aprovação do Comitê de Ética em Pesquisa com o Parecer de oㅜ 3.429.826. Resultados: Na maternidade estudada ocorreram 3859 partos no ano de 2017. A maior prevalência foi dos partos eutócicos com um percentual de $73,89 \%$ contra os $28,27 \%$ do parto cesárea, sendo as mulheres jovens a principal faixa etária das cesáreas com $71,44 \%$. As indicações médicas para o parto cesárea $21,72 \%$ foi por iteratividade, totalizando 232 casos de parto por esta indicação. Conclusão: As mudanças na assistência, durante o pré-natal, podem ter influências na escolha final que é o parto, tendo, o profissional enfermeiro, importante papel nas instruções para as gestantes.

Palavras-Chave: Maternidade, Cesárea, Parto.

\begin{abstract}
Objective: To describe the incidence of cesarean deliveries, comparing to normal deliveries performed at a Municipal Maternity Hospital in Porto Velho, Rondônia, in the year 2017. Methods: This is a descriptive and quantitative cross-sectional study, based on records from women submitted to cesarean and normal deliveries. Data were tabulated considering the variables: quantitative of normal and cesarean births, age range of pregnant women and criteria for medical indication for cesarean delivery. The data obtained were analyzed using the Excel 2016 Software, where the results were expressed through tables and graphs. The research was approved by the Research Ethics Committee with Opinion No. 3,429,826. Results: In the maternity study, 3859 births occurred in 2017. The highest prevalence was normal delivery, with a percentage of $73.89 \%$ versus $28.27 \%$ of cesarean delivery, with young women being the main age group of cesareans with $71,44 \%$. The medical indications for cesarean section $21.72 \%$ were by iterativity, totaling 232 cases of delivery for this indication. Conclusion: Changes in care during prenatal care may have an influence on the final choice of childbirth, with nurses having an important role in the instructions for pregnant women.
\end{abstract}

Key words: Maternity, Caesarean, Childbirth.

${ }^{1}$ União das Escolas Superiores de Rondônia (UNIRON), Porto Velho-RO.

2 Centro Universitário São Lucas (UniSL), Porto Velho-RO.

*E-mail: jessica.nauama@outlook.com 


\section{RESUMEN}

Objetivo: Describir la incidencia de partos por cesárea en comparación con los partos eutócicos en una Maternidad Municipal de Porto Velho Rondônia en el año 2017. Métodos: Se trata de una investigación descriptiva transversal y cuantitativo, de los registros de las mujeres objeto de Cesáreas y partos eutócicos. Los datos se tabularon teniendo en cuenta las siguientes variables: cuantitativo del parto por cesárea y cesárea, grupo de edad de las mujeres embarazadas y criterios de indicación médica para la cesárea. Los datos obtenidos se analizaron utilizando el software Excel 2016, donde los resultados se expresaron a través de tablas y gráficos. La investigación fue aprobada por el Comité de Ética en Investigación con Opinión No. 3,429,826. Resultados: En la maternidad estudiada, hubo 3859 nacimientos en el año 2017. La prevalencia más alta fue de partos eutocicos, con un porcentaje del $73.89 \%$ contra $28.27 \%$ de los partos por cesárea, siendo las mujeres jóvenes el grupo de edad principal de cesáreas con $71,44 \%$. Las indicaciones médicas para la cesárea del $21,72 \%$ fueron por iteratividad, con un total de 232 casos de parto para esta indicación. Conclusión: los cambios en la atención durante la atención prenatal pueden influir en la elección final del parto, y las enfermeras desempeñan un papel importante en las instrucciones para las mujeres embarazadas.

Palabras clave: La Maternidad, Cesárea, El Parto.

\section{INTRODUÇÃO}

A assistência ao parto era feita por parteiras num tempo em que exerciam a atividade sem conhecimento cientifico, uma assistência com base no empirismo e conhecimento popular e aqueles passados de geração em geração. Nesse cenário, a mulher tinha a principal atribuição em seu trabalho de parto e as técnicas realizadas no corpo da mulher aconteciam em sua própria residência, onde elas revelavam seus próprios limites fisiológicos e recusavam a presença masculina (GOMES ARMG, et al., 2014).

A história da cesariana no Brasil se deu no século XX onde as mulheres passaram a ser hospitazadas para que parissem em centros médicos e hospitais, com isso fomentou o uso de medicalização durante 0 trabalho de parto e pós-parto. Com essa nova perspectiva de assistência, a parturiente passou a ser destituída da sua liberdade e perdendo sua autonomia no seu direito de escolha em relação a sua privacidade (GOMES ARMG, et al., 2014).

Com a chegada dos cirurgiões obstétricos, o mecanismo relacionado ao parto surgiu e consequentemente distanciou as parteiras das suas funções. Por anos foi afirmado que a posição ideal para dar à luz era sob uma cama, privada de seus movimentos e deitada, isso facilitava a inserção das drogas e instrumentais cirúrgicos, ou seja, a retirada do apoio familiar e privação da liberdade favorece o desrespeito à parturiente (CUNHA ICBC, et al., 2017).

No Brasil desde a década de 1980, a realidade do parto nos hospitais tem se tornado cada vez mais intervencionista. Os profissionais de saúde têm executado técnicas desnecessárias que geram uma ameaça ao binômio e que podem suceder em complicações posteriores, como: infecções, hemorragias, prematuridade, dentre outros (BARROS LP, et al., 2015).

O parto cesárea é um procedimento que envolve risco de morte materna dez (10) vezes maior quando comparado ao parto normal (BARROS LP, et al., 2015). Os principais riscos para a parturiente estão relacionados a hemorragias, infecções puerperais, embolia pulmonar, complicações anestésicas e morte. Já no caso dos recém-nascidos, há maior risco do parto cesárea resultar em problemas respiratórios, icterícia fisiológica, prematuridade iatrogênica, anóxia, mortalidade neonatal, além de aumentar o risco de morbidade respiratória leve e grave a medida que diminui a idade gestacional e aumenta também o risco de internação em Unidade de Terapia Intensiva (ANJOS CS, et al., 2014).

O índice recomendado pela Organização Mundial de Saúde (OMS), para partos cesarianas, é de 15\%, no entanto, o Brasil possui uma taxa de 55,6\%. Este dado faz do Brasil o segundo colocado em partos cesarianas, perdendo, apenas, para República Dominicana, já considerado uma epidemia no país e traz 
sérios riscos para a saúde da mulher e do recém-nascido, estando diretamente interligado com o aumento da morbi-mortalidade materna e neonatal (RIBEIRO LB, 2016).

É evidente a urgência em ser reduzir os altos índices das taxas de partos cesáreas. O que deve de fato acontecer é o real envolvimento dos diversos atores sociais responsáveis por definir as políticas públicas que ajudariam a colocar critérios para execução de parto cesárea garantindo, à gestante e ao recém-nascido, 0 atendimento integral e humanizado (ANJOS CS, et al., 2014).

O presente estudo tem como objetivo descrever a incidência de partos cesárea, comparando aos partos eutócicos realizados em uma Maternidade Municipal de Porto Velho Rondônia no ano de 2017.

\section{MÉTODOS}

Trata-se de uma pesquisa descritiva e quantitativa de caráter transversal, do tipo documental, realizada na Maternidade Municipal Mãe Esperança que atende como referência em parto humanizado caracterizado como baixo risco / risco habitual em Porto Velho, Rondônia. A população desta pesquisa foi formada por mulheres submetidas aos partos cesáreas e eutócicos no ano de 2017.

A amostra da pesquisa foi constituída através de registros estatísticos existentes na instituição a respeito do quantitativo de mulheres que foram submetidas ao parto cesárea e normal. Sabe-se que esta instituição de saúde registra os partos eutócicos e cesarianas em um banco de dados de forma rigorosa.

Os dados foram coletados considerando as seguintes variáveis: Quantitativo de partos eutócicos e cesarianas, a faixa etária das gestantes e quais os critérios de indicação médica para o parto cesárea. Os dados foram quantificados e procedemos a análise dos dados considerando, como procedimentos de tratamento para a pesquisa, as informações necessárias supracitadas.

Os dados foram analisados mediante utilização do programa Software Excel 2016, onde inicialmente foi elaborado um banco de dados com as informações coletadas, seguida da análise estatística onde os resultados foram expressos por meio de tabela e gráficos.

A presente pesquisa teve aprovação do Comitê de Ética em Pesquisa do Centro Universitário União Educacional do Norte Ltda (UNINORTE) com o CAAE de $n^{\circ}=10215119.4 .0000 .8028$ e Parecer de $n^{*}$ 3.429.826.

\section{RESULTADOS}

Entre o período de janeiro a dezembro de 2017, ocorreram 3.859 partos na maternidade municipal do município de Porto Velho-RO, constatando-se que 1.068 tratavam-se de partos cesáreas, o que corresponde um percentual de $28,27 \%$. Já os partos eutócicos foram 2.791 , o que corresponde a $73,89 \%$ do total de partos realizados no período estudado (Tabela 1).

Quanto ao perfil das parturientes que foram submetidas ao parto cesárea, tratavam-se de mulheres jovens com idade entre 18 a 30 anos sendo um percentual de $71,44 \%$, seguido por $21,44 \%$ de mulheres que estavam na faixa etária entre 31 a 43 anos, e o menor percentil foi de $7,12 \%$ destinado às mulheres entre 14 a 17 anos.

Tabela 1 - Informações relacionadas ao quantitativo de partos eutócicos e partos cesarianos na Maternidade Municipal Mãe Esperança no ano de 2017 em Porto Velho- RO.

\begin{tabular}{lcc}
\hline VARIÁVEL & $\mathbf{N}^{\circ}$ & $\%$ \\
\hline Partos Eutócicos & 2.791 & 73,89 \\
Partos Cesárea & 1.068 & 28,27 \\
TOTAL & $\mathbf{3 . 8 5 9}$ & $\mathbf{1 0 0 , 0}$ \\
\hline
\end{tabular}

Fonte: Dados da Pesquisa, 2017/ N: número; \%: porcentagem. 
No que se refere às indicações médicas para o parto cesárea, 21,72\% foi por Iteratividade, ou seja, 232 casos de indicações por esse tipo de parto, o que reflete a tendência observada não só no Brasil, mas no mundo.

No que se refere a idade das parturientes, o gráfico 1 expressa os percentis da totalidade dos partos realizados no ano de 2017, em que se evidencia o percentual de parto cesárea como a maior predominância entre as mulheres na faixa etária de 18 a 30 anos, correspondendo a $71,44 \%$.

Gráfico 1 - Percentual de faixa etária das gestantes, submetidas à cesárea, atendidas no período de janeiro a dezembro de 2017 na Maternidade Municipal Mãe Esperança em Porto Velho-RO.

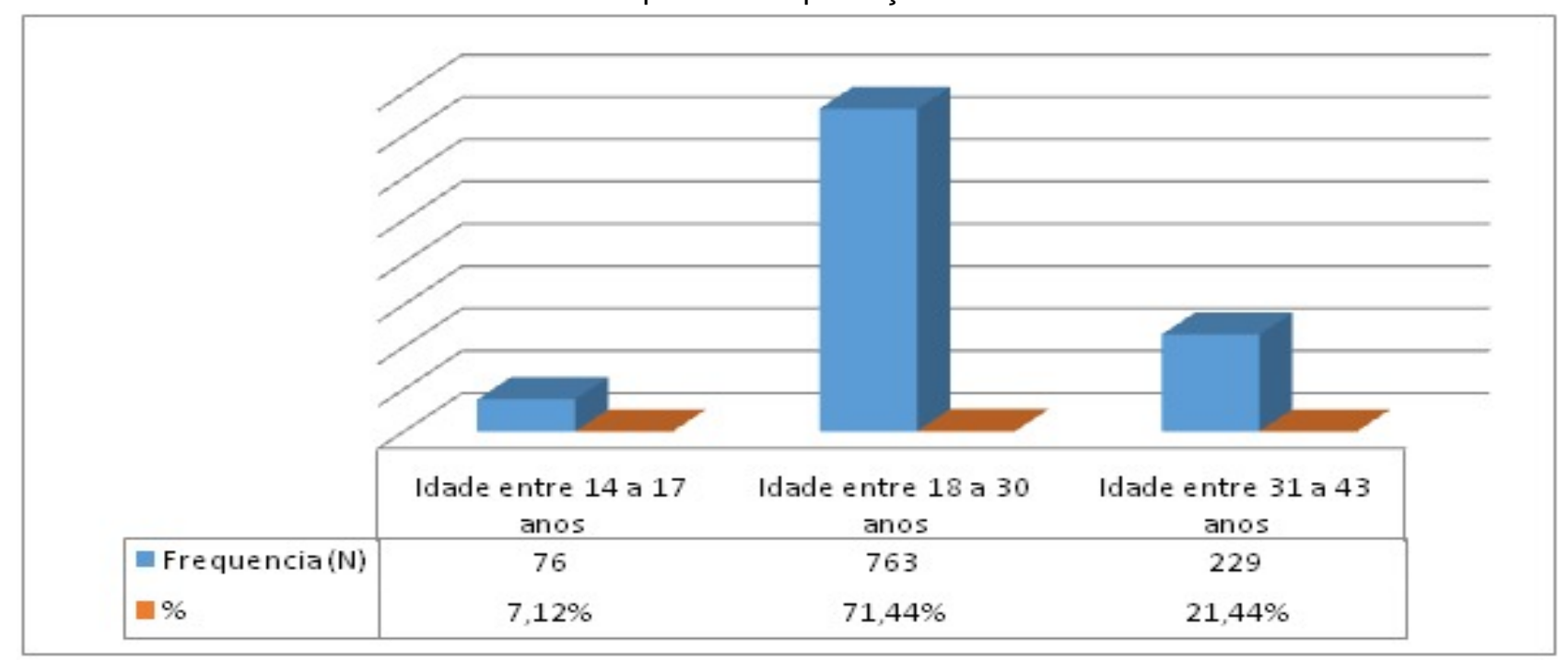

Fonte: Dados da Pesquisa, 2017/ N: número; \%: porcentagem.

Gráfico 2 - Percentual de indicação médica para o parto cesárea na Maternidade Municipal Mãe Esperança no ano de 2017 em Porto Velho - RO.

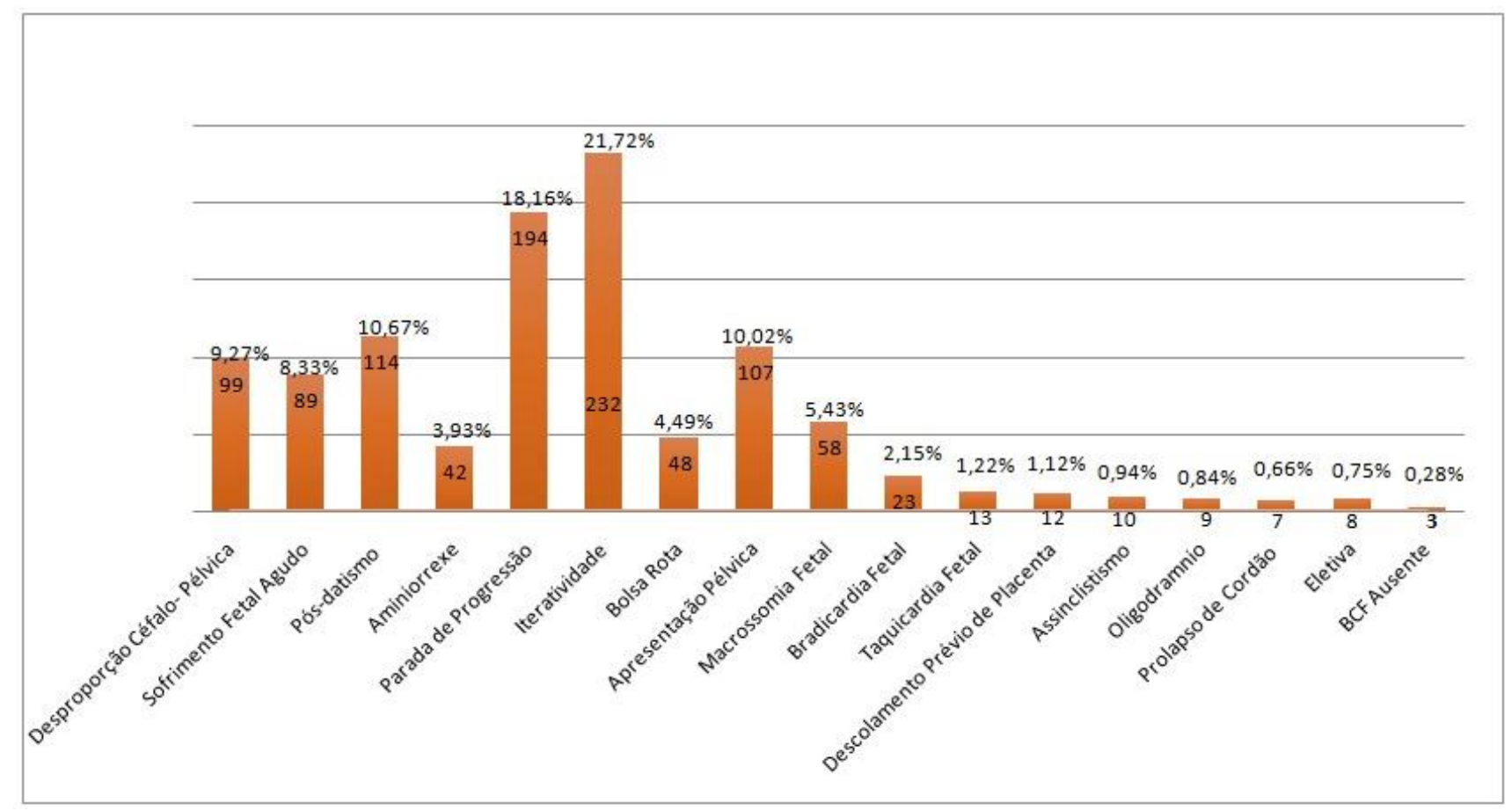

Fonte: Dados da Pesquisa, 2017/ N: número; \%: porcentagem. 
Observa-se que, ao percentual das indicações médicas para o parto cesárea, 21,72\% foi por Iteratividade, ou seja, 232 casos de indicações por esse tipo de parto. Em segundo lugar, com 18,16\%, foi por Parada de Progressão com 194 casos, seguido de 10,67\% por Pós-Datismo com 114 indicações. 10,2\% por Apresentação Pélvica, sendo 107 casos, e 9,27\% por Desproporção Céfalo-Pélvica com 99 registros. 8,33\% por Sofrimento Fetal Agudo com 89 casos, seguidos de 5,43\% por Macrossomia Fetal com 58 casos. 4,49\% por Bolsa Rota com 48 registros, seguido de 3,93\% com 42 casos de Amniorrexe e 2,15\% com 23 registros de Bradicardia Fetal. 1,22\% por Taquicardia Fetal com 13 casos e 1,12\% por Descolamento Prévio de Placenta com 12 registros. 0,94\% com 10 casos de Assinclitismo, 0,84\% com 9 registros de Oligodramnio seguido de $0,75 \%$ sendo eletiva com 8 casos. $0,66 \%$ com 7 registros de Prolapso de Cordão e, por fim, $0,28 \%$ por Batimento Cardíacos Fetais Ausentes, com 3 registros.

Do ponto de vista ético, o princípio filantrópico implica a responsabilidade dos clínicos em minimizar os riscos e maximizar os benefícios. Isto induziria a não efetivação de cesariana sem indicações apropriadas. Contudo, o parto vaginal também pode acarretar consequências, o que implica um balanço entre os dois procedimentos. O princípio de autonomia pode constituir um desafio se a opinião do obstetra e da grávida for dissidente. O princípio de justiça distributiva assenta na correta alocação de recursos e na não discriminação (OLIVA CMPS, et al., 2011).

\section{DISCUSSÃO}

Em um estudo realizado em 2015 na maternidade pública da Região Metropolitana de Belo Horizonte MG, os pesquisadores analisaram dados de 82 parturientes e compararam os números entre partos cesárea e eutócicos, além das variáveis aplicadas ao parto, verificando que, das 82 parturientes, houve 52 casos de cesárea, correspondendo a $63,41 \%$ do número total, enquanto que $36,59 \%$ das mulheres passaram pelo parto eutócico, o que corresponde a 30 casos. A respeito das variáveis, verificou-se que a maior indicação para cesariana foram casos por Desproporção Céfalo-Pélvica (DCP), sendo 42,31\% (MOREIRA BR, et al., 2016).

No entanto, no presente estudo, foi encontrado que houve uma maior prevalência de partos eutócicos. Do total de 3.859 partos realizados no ano de $2017,73,89 \%$ das gestantes optaram pela via vaginal, enquanto que a prevalência da variável entre os partos cesáreas foi de Iteratividade, sendo $21,72 \%$. Isso demonstra que a valorização da cultura dos tempos antigos tem vindo à tona nos tempos atuais. Domingues RMSM et al. (2014) afirmam que o parto vaginal é preferível entre as gestantes por conta de suas vantagens, como a recuperação mais rápida e fácil, e experiências anteriores bem-sucedidas.

Em outro estudo, Kottwitz $\mathrm{F}$ et al. (2018) nos dizem que a escolha pelo parto vaginal está intimamente ligada à recuperação pós-parto, diferentemente do parto cesárea onde as mulheres costumam atribuir, ao pós-parto, dores e um maior tempo de recuperação. O mesmo autor nos diz que para diminuir os índices de parto cesárea é preciso descontruir a ideia da dor e de sofrimento durante o parto, onde os profissionais de saúde possuem um importante papel em explicar a fisiologia do parto e até mesmo os mecanismos da dor para estas gestantes. A Tabela 1 mostra justamente os índices elevados de parto cesárea, no ano de 2017, comparado à recomendação dos índices pela OMS de 10 a 15\%.

O apoio psicológico também é de extrema importância na redução dos índices de cesárea, pois algumas mulheres que já passaram pelo parto vaginal tiveram experiência negativas e acabam optando pela cesárea por medo da experiência se repetir, no entanto, cabe aos profissionais de saúde, durante o pré-natal, fazer este acompanhamento e sempre falar dos benefícios do parto vaginal para a mãe e bebê, e até mesmo desmistificar histórias, como alterações na vida sexual após o parto (DOMINGUES RMSM, et al, 2014). Vemos que, apesar do parto cesárea surgir como um importante procedimento na redução de complicações obstétricas específicas, sendo preciso interromper a gravidez para preservar a vida materna ou fetal, não há redução sistemática e contínua da morbidade materno-infantil com o aumento dos índices de parto cesárea (NOVO JLVG, et al., 2017).

A iniciativa de realizar um parto cesárea por um médico, no lugar de um parto eutócico, pode não parecer ter nenhum efeito econômico, no entanto os resultados dessas decisões podem afetar, seriamente, a 
assistência à saúde da mãe e de seu filho. Esse desperdício de recursos, muitas vezes já escassos, é condenável em razão das deficiências na assistência pré-natal básica, dos baixos índices de diagnósticos preventivos e das deficiências em várias outras necessidades básicas de saúde (OLIVEIRA Q, et al., 2015).

Para Santos GHN et al. (2009), a via de parto, levando-se em consideração a idade maternal, sempre foi uma questão polêmica baseada no preconceito de que as adolescentes não estivessem "prontas" para dar à luz por parto vaginal, com bacia e musculatura uterina imatura para a parturição e por despreparo emocional, no entanto, os dados da literatura mostram exatamente o contrário, onde o desempenho obstétrico das adolescentes é igual ou até melhor que o das mulheres adultas quanto à via de parto.

Considerando esse cenário, é primordial planejar e programar políticas públicas que possam contemplar essas diferenças regionais e os fatores associados às cesarianas. Também é importante enfatizar a inclusão de ações educativas para os profissionais de saúde que atuam na área obstétrica, a fim de melhorar as habilidades de acolhimento, a assistência da mãe e do bebê e reduzir o número de cesarianas desnecessariamente realizadas (EUFRÁSIO LS, et al., 2018).

Eufrásio LS et al. (2018) corroboram esse pensamento definindo que não conseguimos determinar se as cesáreas mostravam uma indicação real para cada mulher da amostra e para cada um de seus partos. Sugerimos para estudos futuros a investigação dos dados, bem como a avaliação da peculiaridade do prénatal e parto, além de outras variáveis associadas ao parto, como vínculo mãe-bebê, saúde mental da mulher e desfechos pediátricos, pois também podem interferir na incidência de cesárea.

\section{CONCLUSÃO}

Através da coleta de dados, foi possível descrever sobre os procedimentos realizados na maternidade para os partos cesáreas e eutócicos, possibilitando a comparação entre eles e sua análise quantitativa. Consideramos que este estudo avança os dados ao quantificar a evidência do parto cesárea em mulheres de Porto - Velho, Rondônia. Por fim, acreditamos que as mudanças na assistência, durante o pré-natal, podem ter influências na escolha final que é o parto. Na Atenção Básica, o enfermeiro tem um papel fundamental nas instruções durante o pré-natal e devem esclarecer todas as dúvidas da gestante sobre a escolha da via de parto e que elas tenham o total conhecimento acerca das implicações que lhe são dadas.

\section{REFERÊNCIAS}

1. ANJOS CS et al. Enfermagem Obstétrica. Rio de Janeiro, set/dez; 1(3):86-94, 2014.

2. BARROS LP, et al. O parto humanizado e seu impacto na assistência à saúde. Revista educação em saúde. V. 3, n. 2, p. 64-71, 2015.

3. Brasil. Ministério da Saúde. DATASUS. Informações de saúde. Estatísticas vitais. Nascidos vivos [Internet]. Brasília; 2015.

4. CUNHA ICBC et al. Caracterização da rede obstétrica nos serviços de saúde. Revista de enfermagem UFPE online. Recife, v. 11, n. 6, jun., 2017.

5. DOMINGUES RMSM et al. Processo de decisão pelo tipo de parto no Brasil: da preferência inicial das mulheres à via de parto. Cad. Saúde Pública, Rio de Janeiro, 30 Sup: S101-S116, 2014.

6. EUFRASIO LS et al. Diferenças e fatores regionais brasileiros associados à prevalência de cesarianas. Fisioter. mov. Curitiba, v. 31, e003108, 2018.

7. GOMES ARMG et al. Assistência de enfermagem obstétrica na humanização do parto normal. Revista ciência científica de enfermagem. São Paulo, v. 11, n. 4, p. 23-27, 2014.

8. KOTTWITZ F et al. Via de parto preferida por puérperas e suas motivações. Escola Anna Nery 22(1) 2018.

9. MOREIRA BR et al. Determinantes relacionados ao parto cesáreo em maternidade pública da Região Metropolitana de Belo Horizonte - MG. Rev Med Minas Gerais; 26: e-1799, 2016.

10. NOVO JLVG et al. Indicações de partos cesáreos em hospitais de atendimento ao Sistema Único de Saúde: baixo e alto riscos. Rev Fac Ciênc Méd Sorocaba. 19(2):67-71, 2017.

11. OLIVA CMPS. Cesariana versus Parto Vaginal: como nascer. 2011.

12. OLIVEIRA Q et al. Incidência e características de cesáreas e de partos normais: estudo em uma cidade no interior do Ceará. Revista Brasileira de Enfermagem 2005, 58, 2015.

13. RIBEIRO LB. Nascer em Belo Horizonte: cesarianas desnecessárias e prematuridade. Dissertação (Mestrado em Enfermagem) - Escola de Enfermagem, Universidade Federal de Minas Gerais, Belo Horizonte, 2016.

14. SANTOS GHN et al. Impacto da idade materna sobre os resultados perinatais e via de parto. Rev. Bras. Ginecol. Obstet. [Internet]. July [cited 2019 July 06] ; 31( 7 ): 326-334, 2009. 